Document downloaded from:

http://hdl.handle.net/10251/83651

This paper must be cited as:

Martínez-Zamora, JȦ.; Belenguer, Ȧ.; Esteban González, H.; Boria Esbert, VE. (2016). Segmentation strategy for the efficient analysis and design of substrate integrated waveguide directly coupled cavity filters. IET Microwaves Antennas and Propagation. 10(3):283-287. doi:10.1049/iet-map.2015.0284.

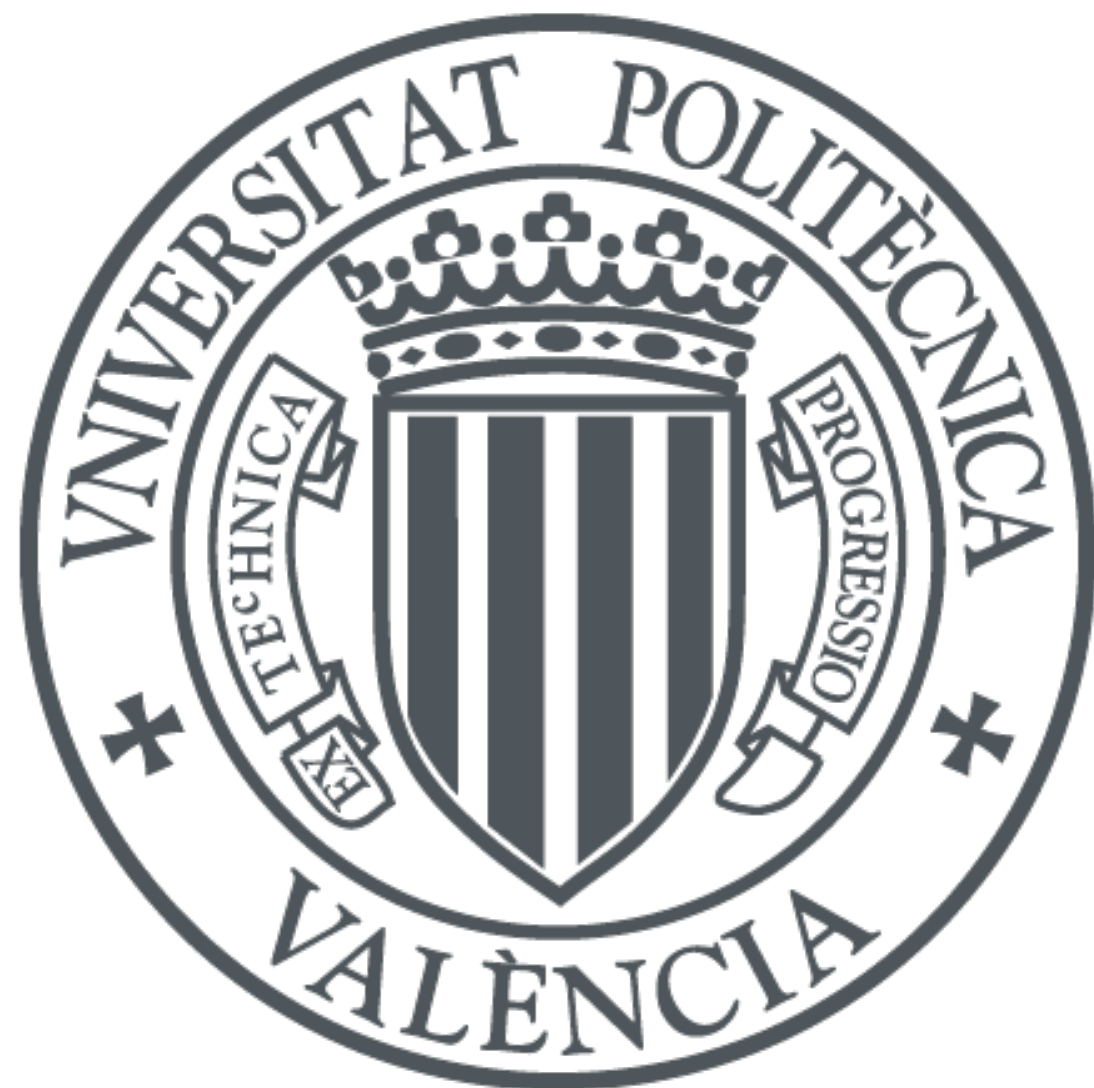

The final publication is available at

http://dx.doi.org/10.1049/iet-map.2015.0284

Copyright Institution of Engineering and Technology (IET)

Additional Information

This paper is a postprint of a paper submitted to and accepted for publication in IET Microwaves Antennas and Propagation and is subject to Institution of Engineering and Technology Copyright. The copy of record is available at IET Digital Library 


\title{
Segmentation Strategy for the Efficient Analysis and Design of Substrate Integrated Waveguide Directly Coupled Cavity Filters
}

\author{
Juan Á. Martínez ${ }^{(1)}$, Ángel Belenguer ${ }^{(1)}$, Senior member, IEEE, Héctor Esteban ${ }^{(2)}$, Senior member, IEEE \\ and Vicente E. Boria ${ }^{(2)}$, Senior Member, IEEE \\ (1) Departamento de Ingeniería Eléctrica, Electrónica, Automática y Comunicaciones \\ Universidad de Castilla-La Mancha, Escuela Politécnica de Cuenca, Campus Universitario, 16071 Cuenca, Spain \\ e-mail: angel.belenguer@uclm.es \\ (2) Departamento de Comunicaciones, Universidad Politécnica de Valencia, 46022 Valencia, Spain \\ e-mail: hesteban@dcom.upv.es
}

\begin{abstract}
In this paper a new segmentation strategy is presented for the full-wave analysis of directly coupled cavity filters in substrate integrated waveguide technology. The whole SIW filter is enclosed inside an external fictitious rectangular waveguide, which does not affect the propagation inside the SIW assuming that the SIW is well designed and there is not significant power leakage. The external rectangular waveguide allows to easily segment the structure into building blocks that are composed of circular metallic vias inside a rectangular waveguide. The GSM of these building blocks are obtained with highly efficient techniques specifically suited for the analysis of H-plane rectangular waveguided devices. Some building blocks are repeated along the structure, and their scattering matrix has to be computed only once, thus improving the numerical efficiency. The scattering matrices of all the building blocks are cascaded using an appropriate method, and the scattering matrix of the whole SIW filter is finally obtained.

In order to test the efficiency of this new method, a SIW filter of 8 coupled cavities with a bandpass response centred at $11 \mathrm{GHz}$ is analysed. Results from this analysis show that the computational time has been significantly reduced when compared with other specific SIW analysis methods or with commercial general purpose software, while maintaining a good accuracy.
\end{abstract}

\section{Index Terms}

Electromagnetic modelling, method of moments, microwave filters, mode matching, substrate integrated waveguides.

\section{INTRODUCTION}

Substrate Integrated Waveguides (SIW) [1]-[3] have proved to be a very good alternative for the implementation of many microwave devices such as couplers [4], filters [5], tunable filters [6], power dividers/combiners [7], [8], circulators [9], attenuators [10], diplexers [11], or antennas [12]-[14].

This new type of transmission line is formed by a dielectric substrate with two rows of metallized via-holes. These metallized holes, together with the upper and lower metallic layers of the substrate, form a synthesized waveguide that can be manufactured with standard low-cost printed-circuit tools. So SIW components can be integrated inside a substrate in the same fashion as classical planar circuits, but they present the advantage for resonant circuits that their quality factors are around 10 times greater than for the equivalent filter with planar technology.

Although general purpose commercial electromagnetic simulators (based on Finite Elements, for example) can be used for the analysis of SIW devices, these tools are not suitable for a design process where many simulations are required in order to find the optimum dimensions that fulfil the specifications. So many analysis techniques of

This work was supported by the Ministerio de Ciencia e Innovación, Spanish Goverment, under Research Projects TEC2013-47037-C5-3-R and TEC2013-47037-C5-1-R. 


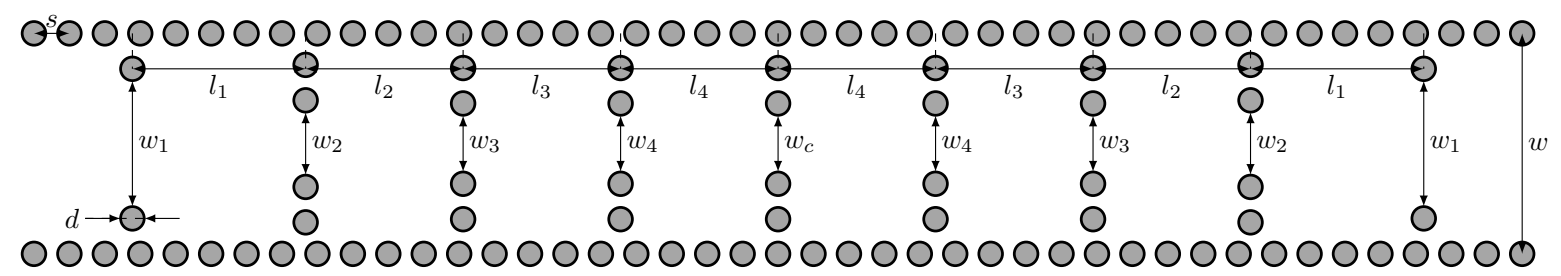

(a) Layout and dimensions

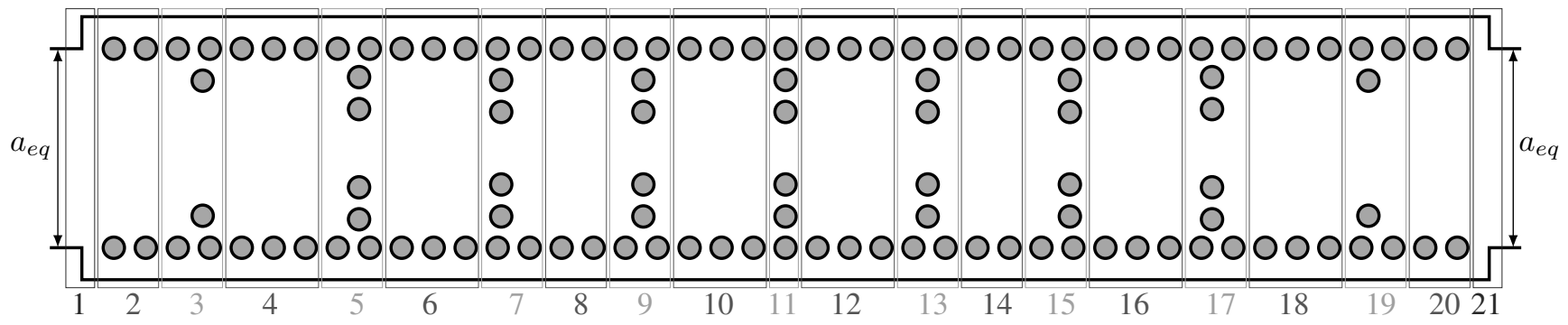

(b) Segmentation of the filter enclosed in an auxiliary rectangular waveguide

Fig. 1. SIW filter of 8 coupled cavities to be analysed

specific purpose for the analysis of SIW structures have been developed and presented in the technical literature, either based on the Boundary Integral-Resonant Mode Expansion method [15], hybrid methods combining Method of Moments and Cylindrical Eigenfunction Expansion [16], hybrid methods combining Method of Moments, Mode Matching and Cylindrical Field Expansions [17], or hybrid methods combining Cylindrical Field Expansion and Mode Matching [18], [19]. However, greater efforts can still be devoted in order to further improve the efficiency of these specific analysis methods.

A possible way to speed up the analysis of a SIW device is to use a segmentation strategy. The segmentation of a SIW device is complex because its transversal section is not constant and therefore the field distribution of the modes propagating inside the SIW structure are not constant along the propagation direction. Direct segmentation of a SIW device would lead to building blocks that should be analysed using open-space analysis methods which are time-consuming, and therefore the reduction of time achieved by the segmentation could be spoiled by the use of time-consuming methods for the analysis of each building block. For that reason some authors have enclosed the SIW device inside an external fictitious rectangular waveguide [20]-[23]. This external waveguide does not affect the propagation inside the SIW, assuming that the SIW is well designed and there is not significant power leakage outside the SIW (this is ensured if the design rules of [24] are observed). The existence of this external rectangular waveguide allows to easily segment the structure into building blocks that are composed now of metallic vias inside a rectangular waveguide. In [20]-[23] a very efficient mode-matching technique is used in order to analyze each building block. However, the metallic vias can only be rectangular (in real SIW structures the vias are circular), and when there are several vias in one of these building blocks they must be aligned, all of them placed in the same position along the propagation direction, which makes it more difficult the design of the SIW device since the position of the vias inside the SIW structure cannot be freely decided. They must be aligned with the vias that form the lateral walls of the SIW.

In this work a different segmentation strategy is used for analysing SIW coupled cavities filters. The purely modal, highly efficient and very accurate technique specific for the analysis of H-plane devices in rectangular waveguides of [25] is used in order to analyze each building block and obtain its Generalized Scattering Matrix (GSM). This method allows the analysis of circular vias (as in real SIW devices), as well as the free positioning of all the vias inside the building block. Some building blocks are repeated along the structure, and their scattering matrix has to be computed only once, thus improving the efficiency. The scattering matrices of all the building blocks are cascaded using an appropriate method (like [26] or [27]) and the scattering matrix of the whole SIW filter is finally obtained.

In order to test the efficiency of this new method, a SIW filter of 8 coupled cavities with a bandpass response centred at $11 \mathrm{GHz}$ is analysed. Results from this analysis show that the computational time has been significantly 
reduced when compared with other specific SIW analysis methods, or with commercial general purpose software, while maintaining a good accuracy.

\section{Segmentation StRategy}

In order to illustrate the segmentation strategy, an 8 coupled cavities filter in SIW technology is going to be considered. The layout of this filter is shown in Figure 1(a). For easily segmenting the filter into simple building blocks, an external rectangular waveguide, as it has already been explained, which is a fictitious and auxiliary waveguide, is going to be used to enclose the SIW structure (see Figure1(b)). As it can be observed, in the input and output accessing ports, steps in the rectangular waveguide are inserted so that the whole structure is accessed with rectangular waveguides whose width $a_{e q}$ is the width of the equivalent waveguide of the SIW line (see [2]). This ensures that the obtained circuital parameters (GSM) are related to the modes of the equivalent waveguide, which are almost identical to the modes of the SIW line.

Therefore, as shown in Figure 1(b), the whole structure can be split into three different types of building blocks. Each type of block is enclosed in a rectangular box depicted with a different intensity of grey (black for the steps between waveguides of different section, dark grey for empty SIW lines, and light grey for the SIW irises).

Next the three types of building blocks resulting from the segmentation of the whole structure are described.

\section{A. Waveguide steps}

The first and the last block (blocks 1 and 21 in Figure 1(b)) are simple steps in rectangular waveguide. The multimodal scattering parameters (GSM) of block 1 can be easily obtained with standard Mode-Matching techniques [28]. Since block 21 is the same as block 1 but changing the order of the ports, the GSM of block 21 is the same as for block 1 but changing the position of some submatrices.

\section{B. Waveguide filled with circular vias}

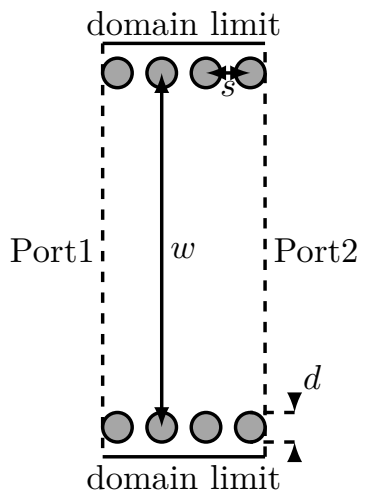

(a)

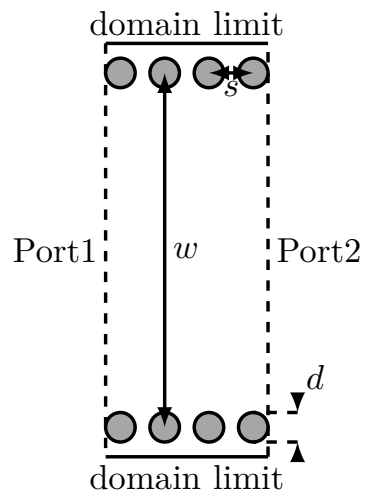

(b)

Fig. 2. Building blocks in which the SIW filter is divided. (a) Empty SIW line block. (b) Iris block.

The other two types of building blocks (SIW empty lines and SIW irises inside the external rectangular waveguide) in which the structure is divided are depicted with more detail in Figure 2. In order to efficiently analyse these building blocks the method of [25] is used. This is a highly efficient and accurate method for the analysis of arbitrary H-plane devices in a rectangular waveguide. This method provides with the GSM of the building block using a discrete frequency sweep. It is very important for the success of the segmentation strategy that the method used to analyse each building block is a highly accurate method, such as the method of [25]. Otherwise, small errors in the computation of the GSM of each building block accumulate when cascading several GSMs, and come up with a significant error in the final GSM of the whole SIW device. It is also important that the method is highly efficient for the segmentation strategy to end up with an important reduction of the overall computational time.

Next the blocks of empty SIW lines and the blocks of SIW irises are described. 
1) Block of empty SIW line: In this block as many pairs of vias as needed are placed in order to model an empty SIW line of a certain length. This type of block appears between the input port and the first iris, between the last iris and the output port, and between each pair of consecutive irises. If the number of vias is the same for two of these blocks, their GMSs are equal and need to be computed only once.

2) Block of SIW iris: This type of block houses a SIW iris. In order to construct the block, the vias of the iris that are closer to the middle of the waveguide (separated a distance of $w_{n}$ in Figure 2(b)) are placed in the first place. Then as many vias as needed are placed between these two initial vias and the lateral walls of the SIW line. The vias that form the iris can be placed at an arbitrary distance $l_{x n}$ (see Figure 2(b)) from the input port. This distance depends on the length of the correspondent resonator cavity $l_{n}$ (see Figure 1(a)).

As already mentioned, in a line filter like the one in Figure 1, there are many blocks that are equal, so their GSMs only has to be computed once. This greatly decreases the total computational time, which is mainly used for cascading the GSMs of all the building blocks, since there are little blocks whose GSM must be calculated.

Another measure to reduce computational time is to compute the GSM of the blocks until we reach the middle of the filter, if the filter is symmetric, since the second half of the filter has the same GSMs. This reduces the computational time by a factor of 2 .

\section{ANALYSIS TOOLS}

The coupled cavities SIW filter has been analysed with different software tools in order to test the efficiency of the segmentation strategy presented in this work. Next we describe these simulation tools.

\section{A. CST Studio Suite}

Two simulations of the filter have been performed using CST (with a Finite Element Method solver). The first simulation is a discrete simulation with 101 points in frequency between $10 \mathrm{GHz}$ and $12 \mathrm{GHz}$. the second simulation is a fast frequency sweep simulation (interpolated) with a maximum of 31 discrete samples. It is considered a closed problem in CST and the vertical dimension is reduced at maximum (this does not affect the solution if losses are not considered).

\section{B. Hybrid MoM and MM method [17].}

This is the method described in [17]. With this method the whole SIW filter (blocks 2 to 20 in Figure 1(b)) is analysed in a single step, without any segmentation. This GSM is next cascaded to the GSMs of blocks 1 and 21 in Figure 1(b). The filter is analysed with 101 discrete frequency points, and it is also analysed using a fast frequency sweep based on Complex Frequency Hopping. In [17] two fast frequency sweep schemes are described, one based on the Asymptotic Wave Expansion (AWE), and the other based in Complex Frequency Hopping (CFH). The first one (based on AWE), is suitable for narrow band responses. In this case, the filter has a $10 \%$ bandwidth, and the AWE technique is not accurate enough, so the CFH technique has been used, although it is not as fast as AWE.

\section{New method with segmentation}

The SIW filter is enclosed in the external waveguide (as it has been done with CST). Next it is segmented as shown in Figure 1(b), and all the GSMs of the basic building blocks have been computed using 31 modes in each accessing port of the external waveguide. The method of [25] is used for the computation of all the building blocks (blocks 2 to 20 in Figure 1(b)) except for the first and last blocks where mode-matching has been used [28]. 101 frequency points are computed (without any fast frequency sweep).

\section{Results}

The validity of this new segmentation strategy has been tested using the new simulation tool for the design of a coupled-cavities filter with 8 resonant cavities (see Figure 1(a)). The filter is designed for a Rogers 4003C substrate $\left(\varepsilon_{r}=3,55\right.$, and height of $1.524 \mathrm{~mm}$ ), and for a central frequency of $11 \mathrm{GHz}$ and a bandwidth of $1 \mathrm{GHz}$. The dimensions for this filter are presented in Table I.

The filter has been analysed using all the analysis tools described in section III, in order to test the accuracy and efficiency of the new proposed segmentation method. 
TABLE I

DIMMENSIONS FOR THE FILTER OF FIG. 1(A)

\begin{tabular}{|l|l|ll|}
\hline$L_{1}=8.2821 \mathrm{~mm}$ & $w=10.8430 \mathrm{~mm}$ & \multicolumn{2}{|l|}{$w_{4}=4.1763 \mathrm{~mm}$} \\
\hline$L_{2}=9.3734 \mathrm{~mm}$ & $w_{1}=6.1832 \mathrm{~mm}$ & $w_{c}=4.1534 \mathrm{~mm}$ \\
\hline$L_{3}=9.6392 \mathrm{~mm}$ & $w_{2}=4.6753 \mathrm{~mm}$ & $s=1.5$ & $\mathrm{~mm}$ \\
\hline$L_{4}=9.6943 \mathrm{~mm}$ & $w_{3}=4.2710 \mathrm{~mm}$ & $d=1$ & $\mathrm{~mm}$ \\
\hline
\end{tabular}

TABLE II

COST COMPARISON

\begin{tabular}{|c|c|c|c|c|}
\hline \multicolumn{2}{|c|}{ CST } & \multicolumn{2}{c|}{ Hybrid MoM-MM [17] } & \multirow{2}{*}{ Segmentation (with [25]) } \\
\hline Discrete & Fast & Discrete & CFH & \\
\hline $655 \mathrm{~s}$ & $267 \mathrm{~s}$ & $313 \mathrm{~s}$ & $129 \mathrm{~s}$ & $34 \mathrm{~s}$ \\
\hline
\end{tabular}

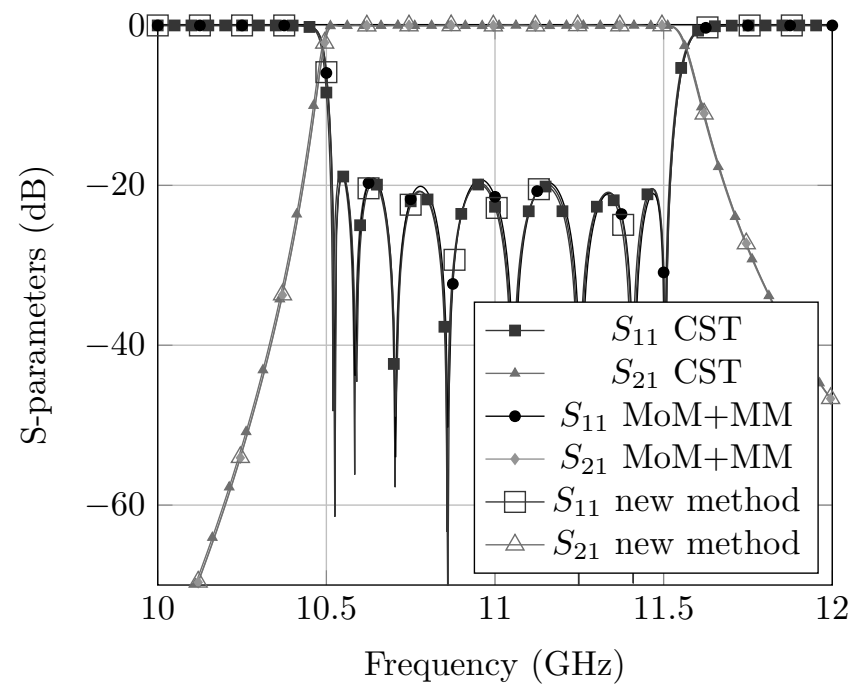

Fig. 3. Analysis of the 8 coupled-cavities SIW filter with the method of [17] (MoM+MM), with CST (FEM solver), and with the new segmentation technique ( [25] and cascade connection)

The accuracy is first tested comparing the scattering parameters of the filters calculated with each one of the analysis tools. Results of this comparison are depicted in Figure 3. It can be observed that all the curves are in excellent agreement, so the accuracy of the new method has been successfully tested.

The efficiency of the segmentation technique is next tested comparing the computational time required to analyse the filter by each one of the analysis tools. The comparison is shown in Table II. As it can be observed, with new method the simulation times is only 34 seconds, whereas with the method of [17] with discrete sweep the simulation time is 313 seconds. This means that the cost of the new method is only the $11 \%$ of the original cost with [17]. With the fast frequency sweep, the method of [17] takes 129 seconds. Comparing with this method the segmentation requires only $26 \%$ of such time. But if some fast frequency sweep scheme was applied to the new segmentation technique, this computational effort could be further reduced. Finally, when compared to CST, the new method takes only $5 \%$ of the time required by CST with discrete sweep, and only $13 \%$ of the time required by CST with a fast frequency sweep scheme. For the CST simulations, the default values in CST 2014 have been used for the initial tetrahedral mesh (4 cells per wavelength in the model and in the background). The mesh has been refined for the central frequency of the passband $(11 \mathrm{GHz})$ with an adaptive mesh scheme with a maximum of 25 passes and a convergence criteria of 0.0025 of S-parameter threshold and 0.001 of portmode $k_{z} / k_{0}$ threshold. This refinement scheme has resulted in 11,007 tetrahedrons for a filter of order 3 and 37,667 tetrahedrons for the filter of order 8 of Figure 1(a). In order to obtain a mesh as reduced as possible, and taking into account that the analysed devices and the fields are invariant in height, the vertical dimension has been reduced so that only a tetrahedron is required to mesh the filter along the vertical dimension. In this case, a filter centered at $11 \mathrm{GHz}$ 


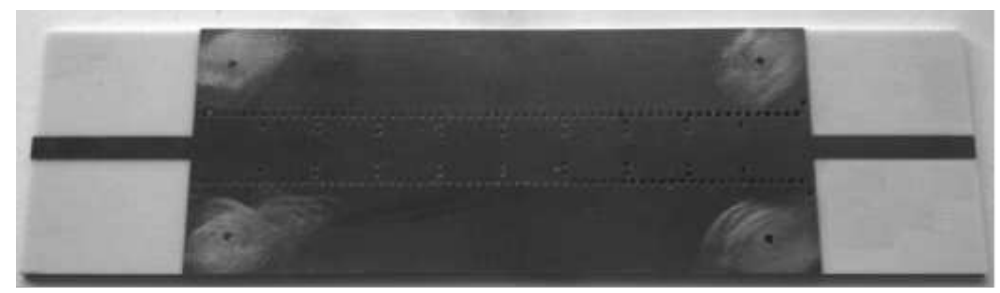

Fig. 4. Manufactured SIW 8 coupled-cavities filter

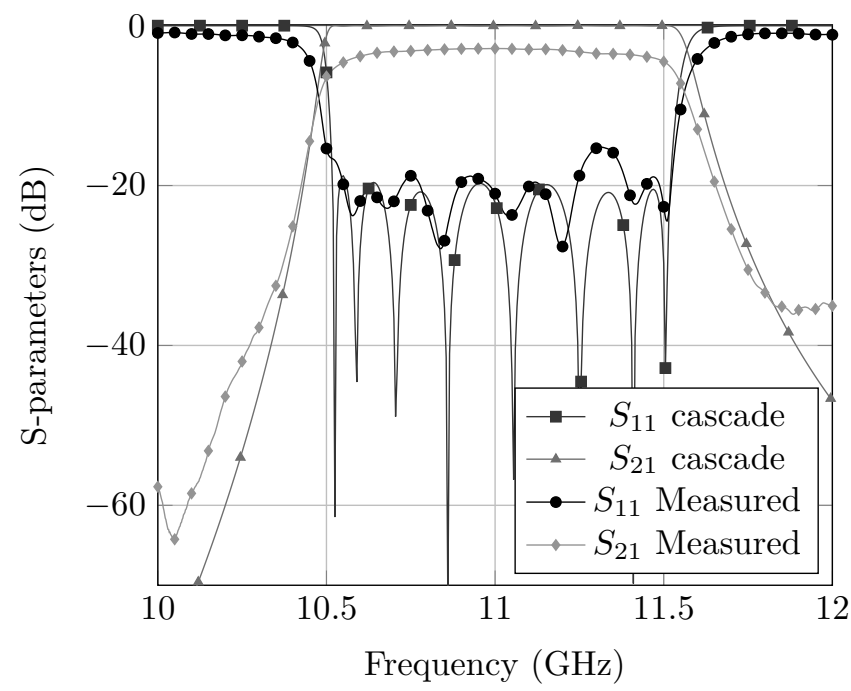

Fig. 5. Comparison between simulation and measurements for the manufactured SIW filter

and designed for a substrate with relative permitivity of 3.55, a vertical dimension of $0.2 \mathrm{~mm}$ accomplishes this objective.

As already has been discussed, the simulation time required by the new method for the filter of Figure 1(a) is 33 seconds. This time is obtained when the scattering matrix of the empty SIW line blocks that appear in the structure more than once are only calculated once, and this scattering matrix is reused. Since the time required for calculating the scattering matrix of an empty SIW line block is reduced, it might be that there is not a significant gain in the overall computational time, and it might not be worth reusing these matrices. So we have simulated without reusing the scattering matrix of repeated elements, but the simulation time when all the scattering matrices are calculated separately is 48 seconds. Therefore there is indeed a significant time reduction thanks to the reuse of the scattering matrices of repeated empty SIW line blocks. Probably this is due to the fact that although the simulation time of the scattering matrix of an empty SIW line is reduced, the computational cost of the remaining parts (other blocks, cascading of matrices) is also quite small.

In order to test that the SIW filter with 8 coupled-cavities has been properly designed, and that the new numeric technique is accurate, the filter has been manufactured and measured with a network analyser. The final manufactured prototype can be seen in Figure 4. The comparison between simulation (without considering losses) and measurement is shown in Figure 5. As it can be appreciated, and neglecting losses, there is a great coincidence between simulation and measurements, so the accuracy of the new simulation tool has been validated.

For a more complete validation, the new method has also been applied for the design of SIW filters with different number of resonant cavities. Table III shows the design time required for the design of each one of the filters (from 3 to 8 resonant cavities) with the new method. The initial point has been obtained using standard techniques for the synthesis of online filters based on the equivalent network with resonators and inverters [29]. The same designs could have been obtained with the other simulators of table II (CST, [17]), but the design time would have been substantially increased more or less in the same proportion as the computation time is increased for one simulation.

In order to test how much the simulation time is increased using other simulators, the final design obtained after 


\begin{tabular}{|c|c|}
\hline Order & Design time (seconds) \\
\hline 3 & $3,114.70$ \\
\hline 4 & $6,990.12$ \\
\hline 5 & $6,433.50$ \\
\hline 6 & $12,273.85$ \\
\hline 7 & $13,660.07$ \\
\hline 8 & $27,453.48$ \\
\hline
\end{tabular}

TABLE III

COMPARISON OF THE DESIGN TIMES FOR FILTERS WITH DIFFERENT NUMBER OF CAVITIES WITH THE NEW METHOD

\begin{tabular}{|c|c|c|c|c|c|}
\hline \multirow{3}{*}{ Order } & \multicolumn{5}{|c|}{ Analysis time (seconds) } \\
\hline & \multicolumn{2}{|c|}{ CST } & \multicolumn{2}{|c|}{ [17] } & \multirow{2}{*}{ New method } \\
\hline & Discrete & Fast & Discrete & CFH & \\
\hline 3 & 215 & 58 & 72.35 & 20.35 & 15.74 \\
\hline 4 & 370 & 94 & 113.50 & 57.88 & 17.93 \\
\hline-5 & 473 & 144 & 161.84 & 68.33 & 18.07 \\
\hline 6 & 485 & 137 & 206.81 & 96.95 & 32.47 \\
\hline 7 & 600 & 205 & 257.05 & 104.44 & 29.23 \\
\hline 8 & 655 & 267 & 313.01 & 128.67 & 33.68 \\
\hline
\end{tabular}

TABLE IV

COMPARISON OF THE SIMULATION TIMES FOR FILTERS WITH DIFFERENT NUMBER OF CAVITIES AND WITH DIFFERENT ANALYSIS METHODS

the optimization process for each filter (from 3 to 8 resonant cavities) has also been simulated with CST and the method of [17]. Results are shown in Table IV and in Figure 6.

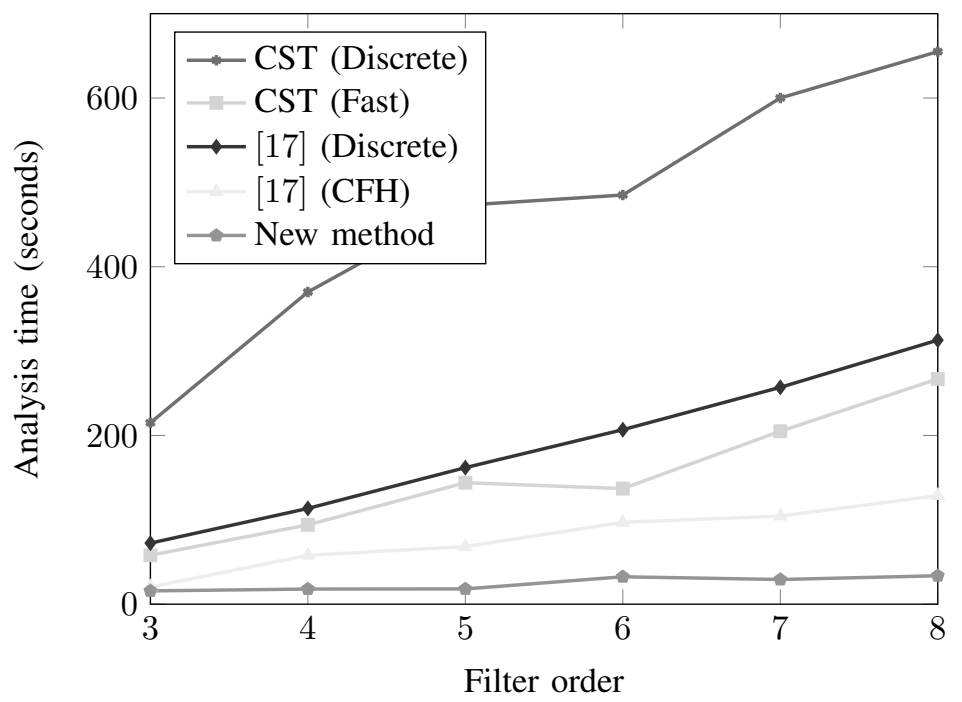

Fig. 6. Comparison of the simulation times for filters with different number of cavities and with different analysis methods

\section{CONCLusions}

A new strategy for the efficient analysis of coupled cavities SIW filters based on segmentation into simple building blocks has been presented.

The accuracy of the method proposed in this work has been validated comparing with CST and with the method of [17] for an 8 coupled cavities SIW filter. The computational time has also been compared, proving the better efficiency of the new method. All simulations were in good agreement. The new method has also been validated by manufacturing and measuring the filter designed for benchmarking. 
The accuracy and efficiency of the new method makes it a good candidate for the design of this type of SIW filters, as well as for other in-line SIW filters where the same segmentation strategy can also be applied.

\section{ACKNOWLEDGEMENT}

This work has been funded by the Ministerio de Economía y Competitividad of the Spanish Government under project number TEC2013-47037-C5-1-R.

\section{REFERENCES}

[1] D. Deslandes and K. Wu, "Integrated microstrip and rectangular waveguide in planar form," IEEE Microw. Wireless Compon. Lett., vol. 11, no. 2, pp. 68-70, Feb. 2001.

[2] Y. Cassivi, L. Perregrini, P. Arcioni, M. Bressan, K. Wu, and G. Conciauro, "Dispersion characteristics of substrate integrated rectangular waveguide," IEEE Microw. Wireless Compon. Lett., vol. 12, no. 9, pp. 333-335, Sept. 2002.

[3] D. Deslandes and K. Wu, "Single-substrate integration technique of planar circuits and waveguide filters," IEEE Trans. Microw. Theory Tech., vol. 51, no. 2, pp. 593-596, Feb. 2003.

[4] J.-X. Chen, W. Hong, Z.-C. Hao, H. Li, and K. Wu, "Development of a low cost microwave mixer using a broad-band substrate integrated waveguide (SIW) coupler," IEEE Microw. Wireless Compon. Lett., vol. 16, no. 2, pp. 84-86, Feb. 2006.

[5] Z.-C. Hao, W. Hong, J.-X. Chen, X.-P. Chen, and K. Wu, "Compact super-wide bandpass substrate integrated waveguide (SIW) filters," IEEE Trans. Microw. Theory Tech., vol. 53, no. 9, pp. 2968-2977, Sept. 2005.

[6] V. Sekar, M. Armendariz, and K. Entesari, "A 1.2-1.6-ghz substrate-integrated-waveguide rf mems tunable filter," Microwave Theory and Techniques, IEEE Transactions on, vol. 59, no. 4, pp. 866-876, April 2011.

[7] J. Hui, W. Feng, and W. Che, "Balun bandpass filter based on multilayer substrate integrated waveguide power divider," Electron. Lett., vol. 48, no. 10, pp. 571-573, May 2012.

[8] T. Djerafi, D. Hammou, S. Tatu, and K. Wu, "Bi-layered substrate integrated waveguide wilkinson power divider/combiner," in IEEE MTT-S International Microwave Symposium Digest (IMS), 2013.

[9] Z. Shi and Z. Shao, "Design of ka-band substrate integrated waveguide circulator," in International Conference on Computational Problem-Solving (ICCP), 2010.

[10] R. F. Xu, A. Farrall, and P. Young, "Analysis of loaded substrate integrated waveguides and attenuators," Microwave and Wireless Components Letters, vol. 24, no. 1, pp. 62-64, 2014.

[11] F. Cheng, X. Lin, K. Song, Y. Jiang, and Y. Fan, "Compact diplexer with high isolation using the dual-mode substrate integrated waveguide resonator," Microwave and Wireless Components Letters, vol. 23, no. 9, pp. 459-461, 2013.

[12] L. Yan, W. Hong, G. Hua, J. Chen, K. Wu, and T. J. Cui, "Simulation and experiment on SIW slot array antennas," IEEE Microw. Wireless Compon. Lett., vol. 14, no. 9, pp. 446-448, Sept. 2004.

[13] M. Henry, C. Free, B. Izqueirdo, J. Batchelor, and P. Young, "Millimeter wave substrate integrated waveguide antennas: Design and fabrication analysis," IEEE Trans. Adv. Packag., vol. 32, no. 1, pp. 93-100, Feb. 2009.

[14] T. Y. Yang, W. Hong, and Y. Zhang, "Wideband millimeter-wave substrate integrated waveguide cavity-backed rectangular patch antenna," IEEE Antennas Wireless Propag. Lett., vol. 13, pp. 205-208, 2014.

[15] M. Bozzi, L. Perregrini, and K. Wu, "Modeling of losses in substrate integrated waveguide by Boundary Integral-Resonant Mode Expansion method," in Microwave Symposium Digest, 2008 IEEE MTT-S International, June 2008, pp. 515 -518.

[16] X. Wu and A. Kishk, "Hybrid of method of moments and cylindrical eigenfunction expansion to study substrate integrated waveguide circuits," IEEE Transactions on Microwave Theory and Techniques, vol. 56, no. 10, pp. 2270 -2276, Oct. 2008.

[17] A. Belenguer, H. Esteban, E. Diaz, C. Bachiller, J. Cascon, and V. E. Boria, "Hybrid technique plus fast frequency sweep for the efficient and accurate analysis of substrate integrated waveguide devices," IEEE Trans. Microw. Theory Tech., vol. 59, no. 3, pp. 552 -560 , Mar. 2011.

[18] E. Diaz Caballero, H. Esteban, A. Belenguer, and V. Boria, "Efficient analysis of substrate integrated waveguide devices using hybrid mode matching between cylindrical and guided modes," Microwave Theory and Techniques, IEEE Transactions on, vol. 60, no. 2, pp. $232-243$, Feb. 2012.

[19] M. Casaletti, R. Sauleau, M. Ettorre, and S. Maci, "Efficient analysis of metallic and dielectric posts in parallel-plate waveguide structures," Microwave Theory and Techniques, IEEE Transactions on, vol. 60, no. 10, pp. 2979 -2989, oct. 2012.

[20] J. Bornemann and F. Taringou, "Substrate-integrated waveguide filter design using mode-matching techniques," in Microwave Conference (EuMC), 2011 41st European, Oct 2011, pp. 1-4.

[21] Z. Kordiboroujeni, F. Taringou, and J. Bornemann, "Efficient mode-matching design of substrate-integrated waveguide filters," in Microwave Conference (EuMC), 2012 42nd European, Oct 2012, pp. 253-256.

[22] Z. Kordiboroujeni and J. Bornemann, "Mode matching design of substrate integrated waveguide diplexers," in Microwave Symposium Digest (IMS), 2013 IEEE MTT-S International, June 2013, pp. 1-3.

[23] Z. Kordiboroujeni, J. Bornemann, and T. Sieverding, "K-band substrate integrated waveguide t-junction diplexer design by modematching techniques," in Microwave Conference (APMC), 2014 Asia-Pacific, Nov 2014, pp. 1297-1299.

[24] F. Xu and K. Wu, "Guided-wave and leakage characteristics of substrate integrated waveguide," IEEE Trans. Microw. Theory Tech., vol. 53, no. 1, pp. 66-73, Jan. 2005.

[25] A. Belenguer, H. Esteban, V. E. Boria, A. L. Borja, and J. Cascon, "Highly efficient heterogeneous modal superposition method for the full-wave analysis of arbitrarily shaped H-plane structures fed through rectangular waveguides," IET Microwaves, Antennas and Propagation, vol. 5, no. 7, pp. 747-755, May 2011.

[26] D. Stock and L. Kaplan, "A comment on the scattering matrix of cascaded 2n-ports (correspondence)," IRE Transactions on Microwave Theory and Techniques, vol. 9, no. 5, pp. 454-454, Sept. 1961. 
[27] A. Belenguer, E. Diaz Caballero, H. Esteban, A. L. Borja, and J. Cascon, "Krylov's solver based technique for the cascade connection of multiple $n$-port multimodal scattering matrices," IEEE Trans. Microw. Theory Tech., vol. 61, no. 2, pp. 720 -726, Feb. 2013.

[28] J. Reiter and F. Arndt, "Rigorous analysis of arbitrarily shaped H- and E-plane discontinuities in rectangular waveguides by a full-wave boundary contour mode-matching method," IEEE Trans. Microw. Theory Tech., vol. 43, no. 4, pp. 796-801, Apr. 1995.

[29] G. L. Matthaei, L. Young, and E. M. T. Jones, Microwave Filters, Impedance-Matching Networks, and Coupling Structures. McGrawHill, 1964.

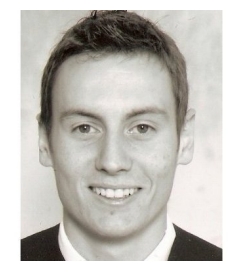

Juan A. Martínez Juan A. Martínez received the Telecommunications Engineering degree from the University of Castilla-La Mancha (UCLM), Cuenca, Spain in 2012. He received the Research Telecommunications (Electronics and Communications specialized) Master Degree from the University Miguel Hernandez (UMH) Elche, Spain in 2013. In 2013 he joined the University of Castilla-La Mancha, where he worked with the Grupo de Electromagnetismo Aplicado as research assistant. His research interests include computational electromagnetics and the analysis and the synthesis of passive microwave circuits and antennas, SIW devices analysis and their applications.

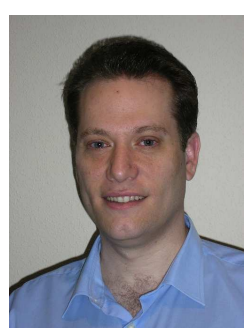

Angel Belenguer (M'04-SM'14) received his degree in telecommunications engineering from the Universidad Politécnica de Valencia (UPV), Spain, in 2000, and his Ph.D. degree, also from the UPV, in 2009. He joined the Universidad de Castilla-La Mancha in 2000, where he is now Profesor Titular de Universidad in the Departamento de Ingenieria Electrica, Electronica, Automatica y Comunicaciones. His research interests include methods in the frequency domain for the full-wave analysis of open-space and guided multiple scattering problems, the application of accelerated solvers or solving strategies (like grouping) to new problems or structures, EM metamaterials, and Substrate Integrated Waveguide (SIW) devices and their applications.

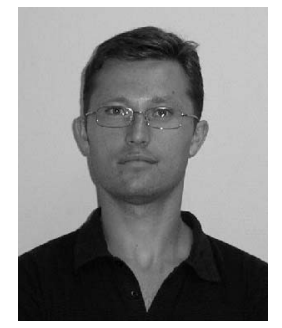

Héctor Esteban González (S'03-M'99-SM'14) received a degree in telecommunications engineering from the Universidad Politécnica de Valencia (UPV), Spain, in 1996, and a Ph.D. degree in 2002. He worked with the Joint Research Centre, European Commission, Ispra, Italy. In 1997, he was with the European Topic Centre on Soil (European Environment Agency). He rejoined the UPV in 1998. His research interests include methods for the full- wave analysis of open-space and guided multiple scattering problems, CAD design of microwave devices, electromagnetic characterization of dielectric and magnetic bodies, and the development, analysis and design of passive devices in substrate integrated transmission lines.

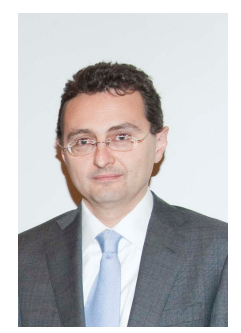

Vicente E. Boria (S'91-A'99-SM'02) was born in Valencia, Spain, on May 18, 1970. He received his Ingeniero de Telecomunicación degree (with first-class honors) and the Doctor Ingeniero de Telecomunicación degree from the Universidad Politécnica de Valencia, Valencia, Spain, in 1993 and 1997, respectively. In 1993 he joined the Departamento de Comunicaciones, Universidad Politécnica de Valencia, where he has been Full Professor since 2003. In 1995 and 1996, he was holding a Spanish Trainee position with the European Space Research and Technology Centre, European Space Agency (ESTEC-ESA), Noordwijk, The Netherlands, where he was involved in the area of EM analysis and design of passive waveguide devices. He has authored or co-authored 7 chapters in technical textbooks, 75 papers in refereed international technical journals, and over 150 papers in international conference proceedings. His current research interests are focused on the analysis and automated design of passive components, left-handed and periodic structures, as well as on the simulation and measurement of power effects in passive waveguide systems. Dr. Boria has been a member of the IEEE Microwave Theory and Techniques Society (IEEE MTT-S) and the IEEE Antennas and Propagation Society (IEEE APS) since 1992. He is member of the Editorial Boards of the IEEE Transactions on Microwave Theory and Techniques, IEEE Microwave and Wireless Components Letters, Proceeding of the IET (Microwaves, Antennas and Propagation), IET Electronics Letters and Radio Science. Since 2013, he serves as Associate Editor of IEEE Microwave and Wireless Components Letters. He is also a member of the Technical Committees of the IEEE-MTT International Microwave Symposium and of the European Microwave Conference. 